\title{
Synthesis of polycyclic and 4,5-diacylthiophene-2-carboxylates via intramolecular Friedel-Crafts alkylations and unusual autooxidative fragmentation of the derivatives obtained from the samarium diiodide-promoted coupling reactions of thiophene-2-carboxylate with carbonyl compounds
}

\author{
Shyh-Ming Yang and Jim-Min Fang* \\ Department of Chemistry, National Taiwan University, Taipei 106, Taiwan
}

Received 11 September 2006; revised 27 November 2006; accepted 28 November 2006

Available online 15 December 2006

\begin{abstract}
Our present study provides an expedient method for the synthesis of novel polycyclic and multi-substituted thiophene derivatives. A series of 4,5-di(hydroxyalkyl)-4,5-dihydrothiophene-2-carboxylates (e.g., 4a-c and 10) were prepared by the SmI $\mathrm{I}_{2}$-promoted three-component coupling reactions of thiophene-2-carboxylate with aromatic aldehydes and 4-methoxyacetophenone. Diol 4a was oxidized by DDQ or pyridinium dichromate to give 5-acyl-4-hydroxyalkyl-4,5-dihydrothiophene-2-carboxylate $\mathbf{6 a}$, which was subjected to dehydration to give either alkene 7 with terminal $\mathrm{C}=\mathrm{C}$ double bond or alkene 15a having conjugation with the ester group, depending on the reaction conditions using different quantities of $p$-toluenesulfonic acid. Alkene $\mathbf{7}$ underwent an intramolecular Friedel-Crafts alkylation to give a tetralone-fused thiophene-2-carboxylate 9. By the similar procedure, a carbazole-fused thiophene $\mathbf{1 4}$ was also prepared. Alkenes 15a-c underwent autooxidative fragmentation to give 4,5-diacylthiophene-2-carboxylates $\mathbf{5 a}-\mathbf{c}$ that were elaborated to pyridazine-fused thiophenes.

(C) 2006 Elsevier Ltd. All rights reserved.
\end{abstract}

\section{Introduction}

Thiophenes and their polycyclic derivatives exhibit remarkable electrochemical, ${ }^{1, a, b}$ optical, ${ }^{1 \mathrm{c}}$ physical, ${ }^{1 \mathrm{~d}}$ and biologi$\mathrm{cal}^{1 \mathrm{e}, \mathrm{f}}$ properties that render their extensive applications in material and pharmaceutical sciences. Though thiophene derivatives have been prepared by various methods, ${ }^{2}$ elaboration of the existing thiophene skeleton with multiple substituents at the desired positions is still a challenging task. To our knowledge, there are only a few reports on the derivatization of thiophene-2-carboxylates. ${ }^{3}$ For example, methyl thiophene-2-carboxylate reacts with paraformaldehyde in the presence of $\mathrm{ZnCl}_{2}$ to give a mixture of 4-chloromethyl-, 5-chloromethyl-, and 4,5-bis(chloromethyl)thiophene-2carboxylate ${ }^{3 a}$ Metalation of thiophene-2-carboxylate and the subsequent electrophilic substitution usually occur at either C-3 or C-5 position; ${ }^{3 b}$ however, introduction of substituents at the $\mathrm{C}-4$ position is still difficult.

Keywords: Samarium diiodide; Coupling reaction; Autooxidation; Fragmentation; Thiophene; Thieno[2,3-d]pyridazine.

* Corresponding author. Tel.: +8862 3366 1663; fax: +8862 2363 7812; e-mail: jmfang@ntu.edu.tw

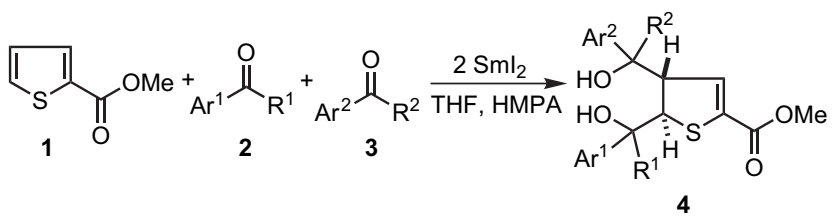

In an approach to elaborate thiophene derivatives at C-4 and C-5 positions, we have utilized $\mathrm{SmI}_{2}$ as the promoter to carry out the tandem double electrophilic reactions of thiophene2-carboxylate 1 with carbonyl compounds (e.g., 2 and $\mathbf{3}$ ) to obtain the three-component coupling products of 4,5-di(hydroxyalkyl)-4,5-dihydrothiophenes 4 in one-pot operation (Eq. 1). ${ }^{4}$ The three-component coupling products prepared as such have been elaborated to the photochromic compounds of 4,5-dialkenylthiophenes ${ }^{4 \mathrm{c}}$ and the sulfurcontaining polyaromatic compounds ${ }^{4 \mathrm{~d}}$ that are applicable to material and biological researches. In order to understand the scope and limitation of this method, we extended this study to synthesize the previously elusive compounds of 4,5-diacylthiophene-2-carboxylates (Fig. 1), and serendipitously observed an unusual acid-catalyzed autooxidative fragmentation reaction during this course of study. 


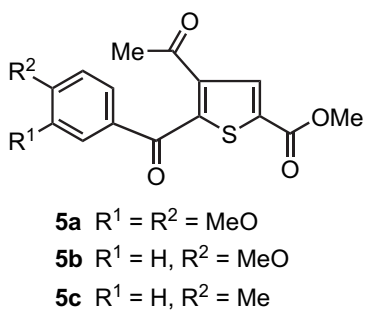

Figure 1. Example of 4,5-diacylthiophene-2-carboxylates that are not reported previously.

\section{Results and discussion}

The coupling reaction of thiophene-2-carboxylate with 3,4-dimethoxybenzaldehyde proceeded smoothly by the promotion of $\mathrm{SmI}_{2}$ (Scheme 1). The intermediate samarium dienolate (A) was trapped by 4-methoxyacetophenone to give diol $\mathbf{4 a}$ in one-pot operation. The intermediate diol $\mathbf{4 a}$, without further purification, was readily converted to ketone 6a by oxidation with DDQ. Ketone 6a was then subjected to an acid-catalyzed dehydration, an oxidative aromatization, and an acid-catalyzed cyclization to afford the desired tetralone-fused thiophene $\mathbf{9}$ via the intermediacy of alkene 7 and thiophene $\mathbf{8}$.

The three-component coupling products $\mathbf{4 b}$ and $\mathbf{4 c}$ were similarly prepared, and then oxidized by DDQ to give the ketone compounds $\mathbf{6 b}$ and $\mathbf{6 c}$. In a recent communication, ${ }^{5}$ we also reported a similar $\mathrm{SmI}_{2}$-promoted coupling reaction of thiophene-2-carboxylate with 1-methylindole-2-carbaldehyde and 4-methoxyacetophenone to afford diol 10 (Scheme 2).
Diols 5a-c and 10 likely have the 4,5-trans configuration that can be established by an attack of the second electrophile on the less hindered face of the dienolate intermediate A (Scheme 1). ${ }^{4}$ The ${ }^{1} \mathrm{H}$ NMR analysis indicated that diol $\mathbf{1 0}$ existed as a mixture of two diastereomers (65:35) differing at the carbinyl centers. Oxidation of diol $\mathbf{1 0}$ (as a diastereomeric mixture) with DDQ at room temperature afforded a single product of ketone $\mathbf{1 1}$. This oxidation reaction was also realized by using pyridinium dichromate (PDC) as the oxidizing agent. No dehydrogenation to aromatic thiophene derivatives occurred on treatment with DDQ or PDC under such mild reaction conditions. Under an atmosphere of nitrogen, alcohol 11 was treated with a catalytic amount of $p$ $\mathrm{TsOH}$ in refluxing benzene to give the dehydration product 12 having a terminal $\mathrm{C}=\mathrm{C}$ double bond, instead of giving the conjugated isomer. The oxidative aromatization of dihydrothiophene $\mathbf{1 2}$ to alkenylthiophene $\mathbf{1 3}$ is realized by using $\mathrm{Pd}(\mathrm{OAc})_{2}$ as the oxidizing agent. The intramolecular Friedel-Crafts alkylation of $\mathbf{1 3}$ is then carried out by the catalysis of $p$-TsOH to give the tetracyclic carbazolothiophene 14. Some carbazole-fused thiophene analogues of 14 exhibiting potent antagonistic activity against the endothelin vasoconstrictor $^{5,6}$ were also prepared by the similar procedures.

Interestingly, we observed that the treatment of $\mathbf{6 a}$ with an increased amount of $p$ - $\mathrm{TsOH}(\sim 0.5$ equiv) in refluxing benzene under an inert atmosphere afforded a $79 \%$ yield of the conjugated compound 15a (Scheme 3), differing from isomer 7 obtained from the acid-catalyzed dehydration (Scheme 1). The $(E)$-configuration in 15a was determined by the ${ }^{1} \mathrm{H}$ NMR analysis, which showed a $12.3 \%$ nuclear Overhauser enhancement of H-5 (at $\delta$ 5.86) upon irradiation of the methyl group at $\delta 1.90$.
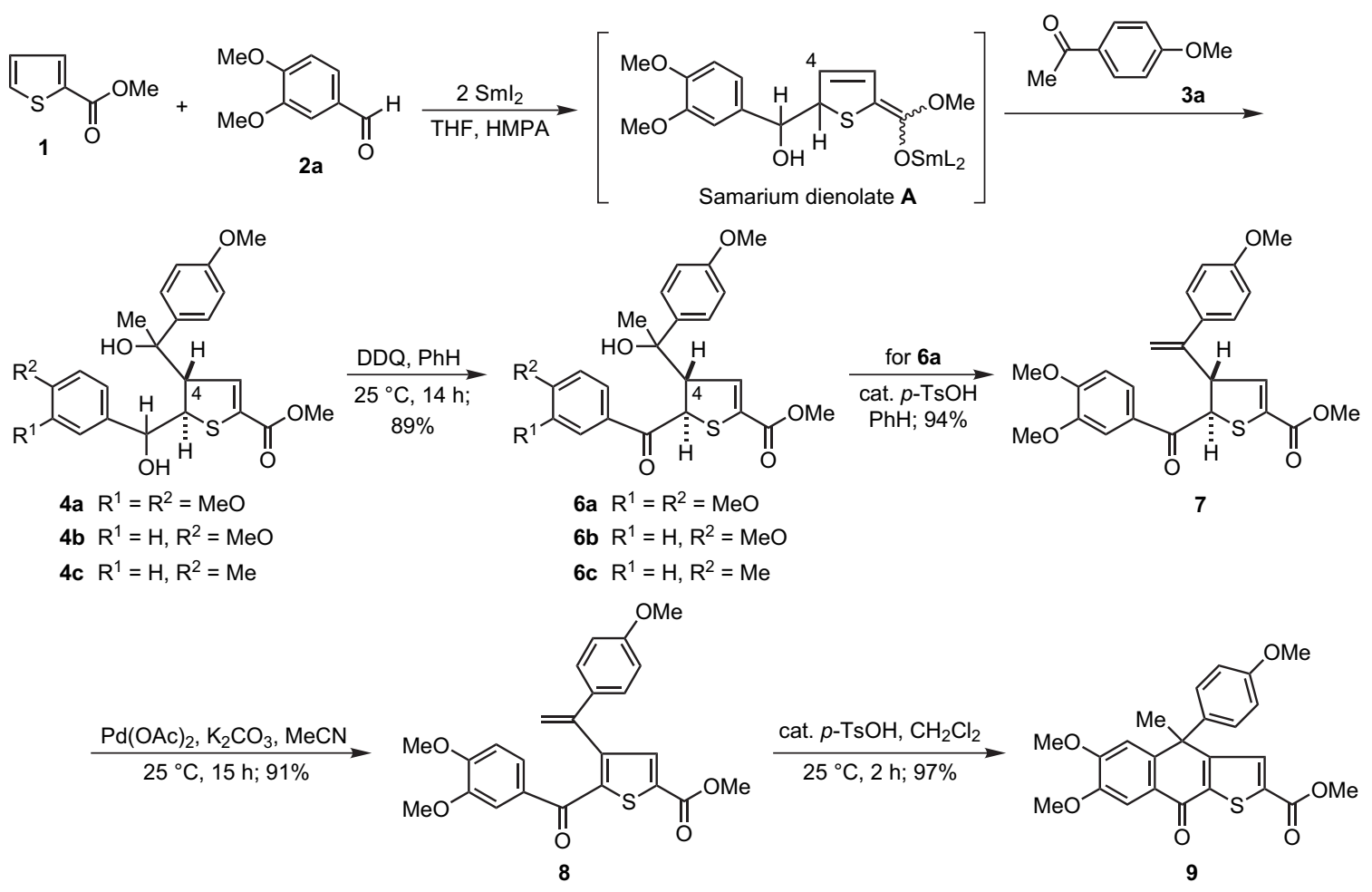


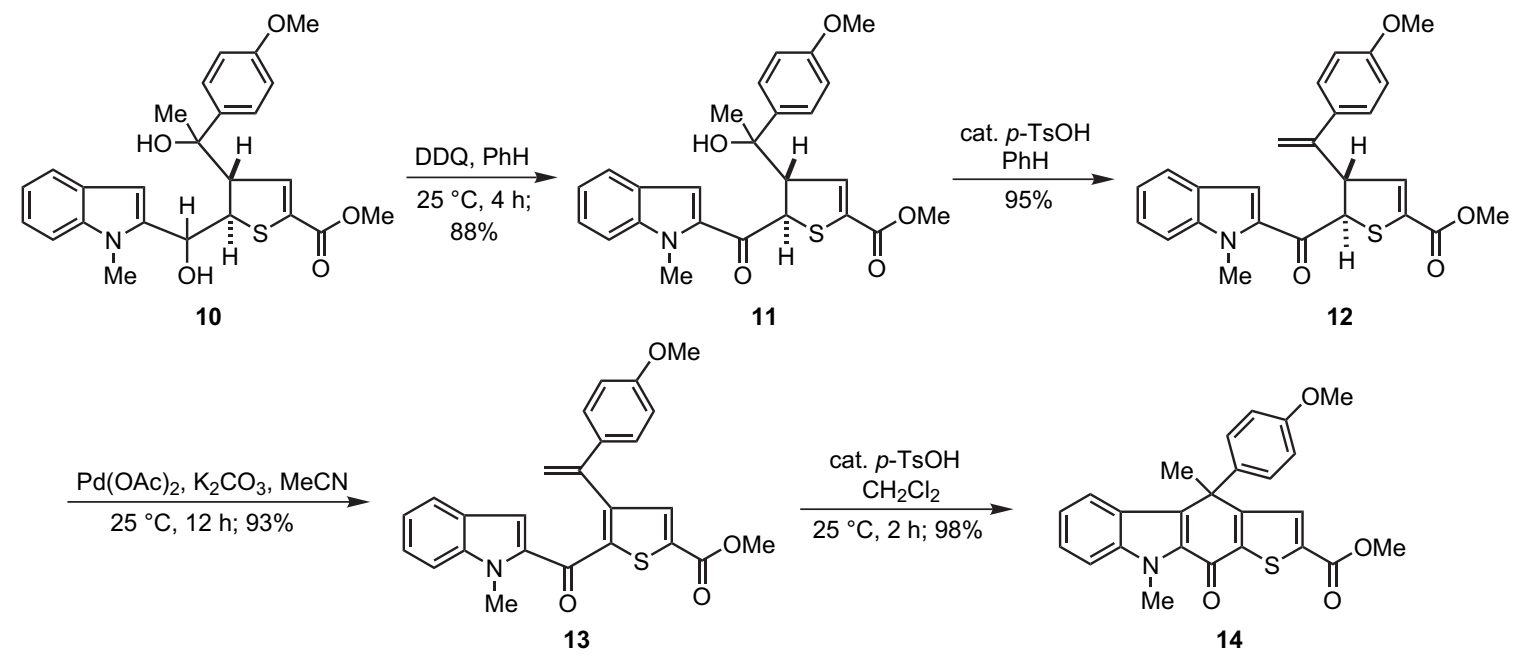

Scheme 2. Synthesis of a carbazole-fused thiophene.
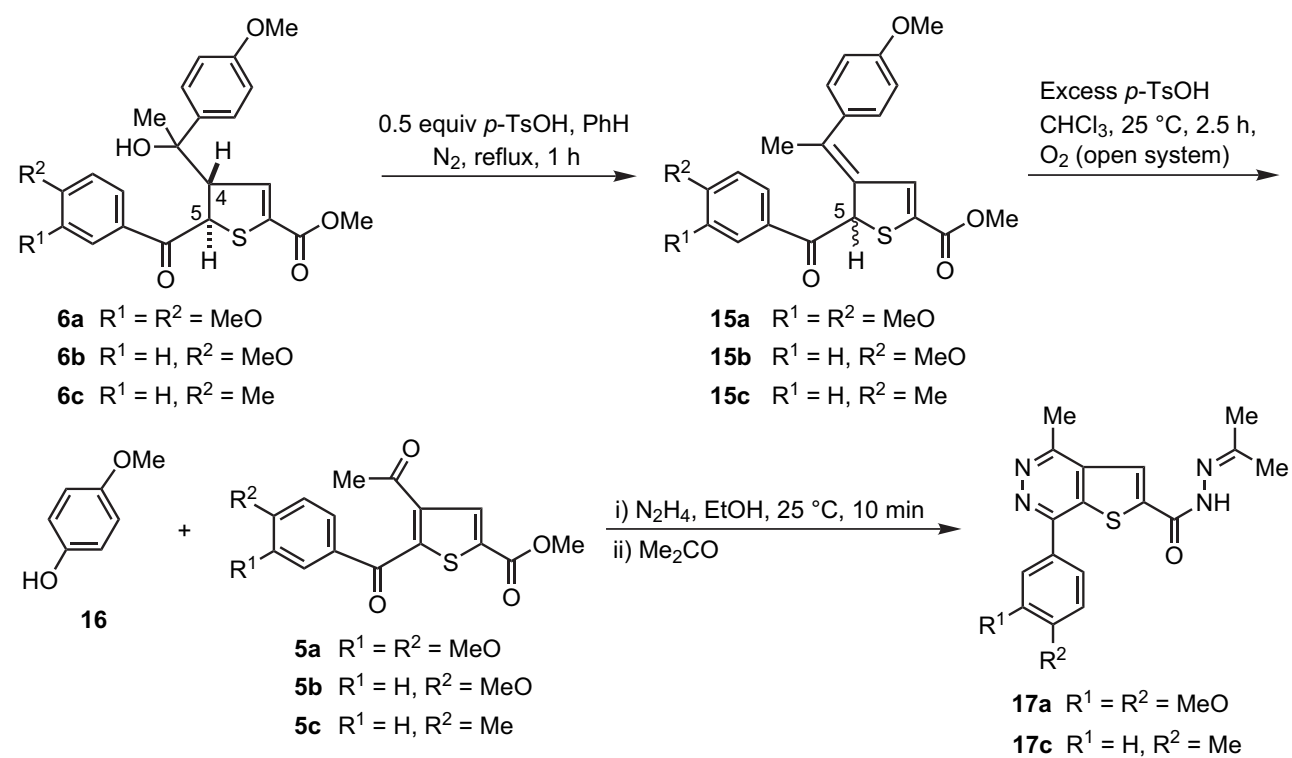

Scheme 3. Formation of 4,5-diacylthiophenes via oxidative fragmentation.

In a serendipitous way, we also found an unusual autooxidation of 15a, giving two oxidative cleavage products $\mathbf{1 6}$ and 5a in quantitative yields (Scheme 3). The autooxidation occurred when a $\mathrm{CHCl}_{3}$ solution of $\mathbf{1 5 a}$ was stirred with stoichiometric amount of $p$-TsOH in air $\left(25^{\circ} \mathrm{C}, 2.5 \mathrm{~h}\right)$. Compound 16 was identified as 4-methoxyphenol, and the structure of 5a was determined to be methyl 4-acetyl5-(3,4-dimethoxybenzoyl)thiophene-2-carboxylate by IR, MS, ${ }^{1} \mathrm{H}$ and ${ }^{13} \mathrm{C}$ NMR spectral analyses. By the similar procedures, compounds $\mathbf{6 b}$ and $\mathbf{6 c}$ were, respectively, treated with $p$-TsOH to give the alkene intermediates $\mathbf{1 5 b}$ and 15c, which underwent autooxidative cleavages in situ to the corresponding 4,5-diacylthiophene-2-carboxylates $\mathbf{5 b}$ and $\mathbf{5 c}$, along with the counterpart of 4-methoxyphenol $\mathbf{1 6}$. The diacylthiophenes $\mathbf{5 a}-\mathbf{c}$ are equipped with the function of 1,4-diketone, which is useful in the construction of various heterocycles. ${ }^{7}$ For example, 5a and $\mathbf{5 c}$ were, respectively, treated with hydrazine, followed by condensation with acetone, to give thieno[2,3- $d]$ pyridazine derivatives 17a and $17 \mathbf{c}$ in quantitative yields. Thieno[2,3- $d]$ pyridazine derivatives have been shown to exhibit anti-inflammatory activity, ${ }^{7 \mathrm{a}}$ and were used as a short-term hypnotic to treat insomnia. ${ }^{7 \mathrm{~b}}$ We also attempted to carry out a straightforward synthesis of 5a-c by the $\mathrm{SmI}_{2}$-promoted double electrophilic reactions of methyl thiophene-2-carboxylate with aromatic aldehyde $\mathrm{Ar}^{1} \mathrm{CHO}$ (or benzoyl chloride) and acetaldehyde (or acetyl chloride). However, all the reactions resulted in complicated mixtures.

Though the real reaction pathways for the dichotomous formation of alkenes $\mathbf{7}$ and 15a from the alcohol 6a were not rigorously determined, we speculated that the dehydration might proceed with E1 mechanism through a stabilized tertiary carbocationic intermediate $\mathbf{C}$ (Scheme 4). The subsequent removal of a proton from the less hindered methyl group would give alkene 7, whereas removal of the internal proton at C-4 would furnish the conjugated compound 15a. Alkene 7 having an isolated $\mathrm{C}=\mathrm{C}$ double bond was partially 

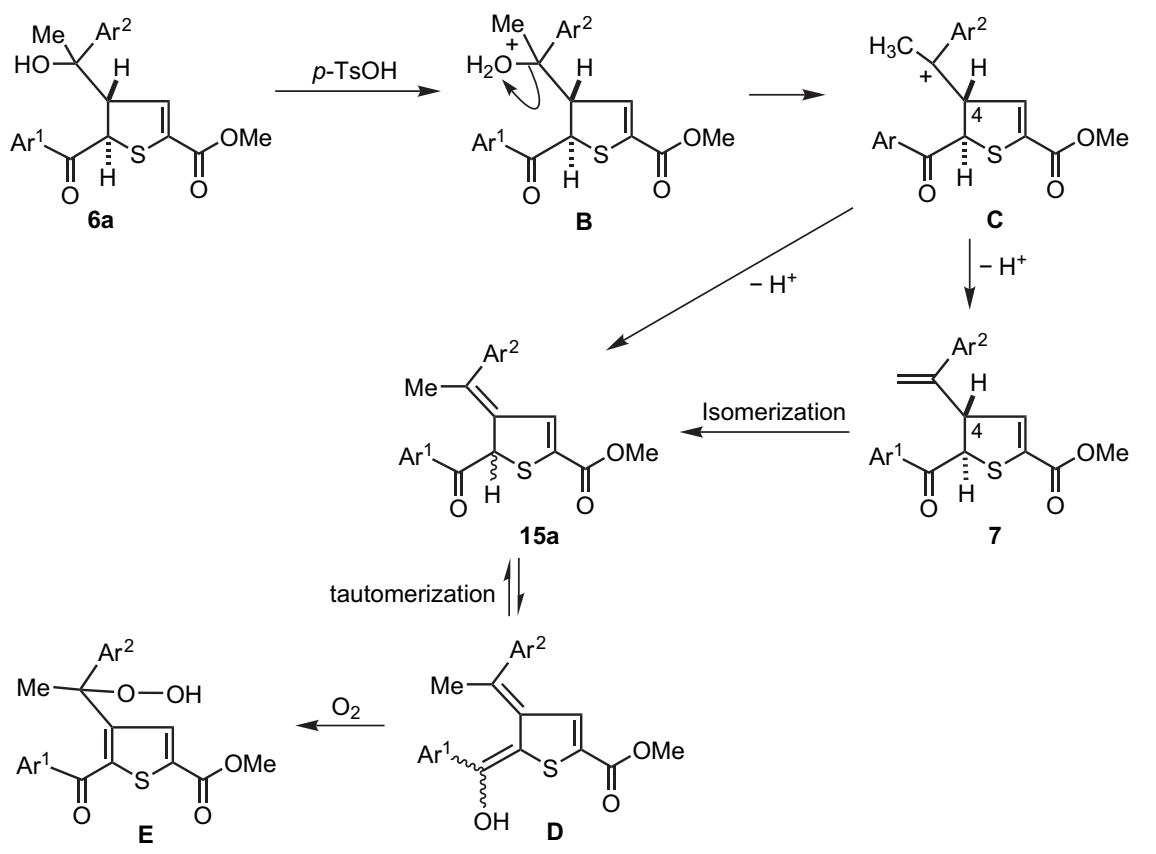<smiles>COC(=O)c1cc(=C(C)C#N)c(=C([15N])O)s1</smiles>

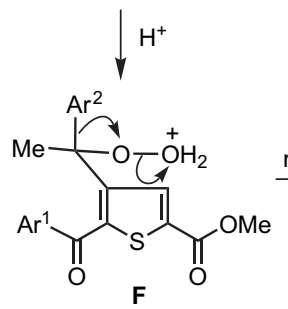
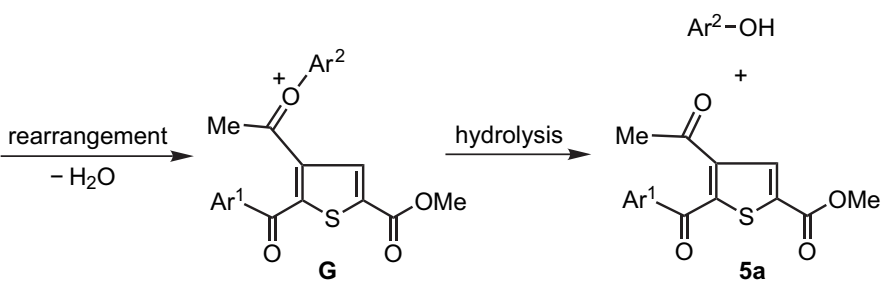

Scheme 4. Putative mechanism for conversion of $6 \mathbf{a}$ to $\mathbf{5 a}$ via dehydration and autooxidative fragmentation.

converted to the conjugated alkene 15a on treatment with $p$-TsOH in refluxing benzene under an atmosphere of nitrogen. We also proposed a possible mechanism for the conversion of $\mathbf{1 5 a}-\mathbf{c}$ to diacylthiophenes $\mathbf{5 a}-\mathbf{c}$ via the fragmentation reaction of a hydroperoxide intermediate. For example, a facilitated enolization of $\mathbf{1 5 a}$ could be effected by a strong acid, $p$ - TsOH (in stoichiometric amount). Upon exposure to air, the dienol intermediate $\mathbf{D}$ would react with dioxygen to give a hydroperoxide intermediate $\mathbf{E}$. The subsequent rearrangement of the hydroperoxide intermediate E would afford 4-methoxyphenol (16, $\left.\mathrm{Ar}^{2}=4-\mathrm{MeOC}_{6} \mathrm{H}_{4}\right)$ and ketone 5a, by analogy to the well-known fragmentation reaction of cumyl hydroperoxide to phenol and acetone. ${ }^{8}$

\section{Conclusion}

We have extended the scope of the previously discovered $\mathrm{SmI}_{2}$-promoted tandem double electrophilic reactions of thiophene-2-carboxylate with aromatic aldehydes and ketones. The three-component coupling products, e.g., 4a and 10, were readily oxidized to the corresponding ketones, e.g., 6a and 11, using DDQ or PDC as the oxidizing agents. In one approach, dehydration of $\mathbf{6 a}$ and $\mathbf{1 1}$ was carried out by using catalytic amount of $p$-TsOH to give alkenes 7 and $\mathbf{1 2}$ with terminal $\mathrm{C}=\mathrm{C}$ double bonds. The alkenes $\mathbf{7}$ and $\mathbf{1 2}$ were subjected to oxidative aromatization and intramolecular Friedel-Crafts alkylation to afford tetralone- and carbazole-fused thiophene-2-carboxylate 9 and 14. In another approach using increased amounts of $p-\mathrm{TsOH}$ ( $\sim 0.5$ equiv), alcohols $\mathbf{6 a}-\mathbf{c}$ were converted to the dehydration products 15a-c, which underwent an unusual autooxidative fragmentation reaction in air to give very high yields of 4,5-diacylthiophene-2-carboxylates. Our present study thus provides an expedient method for the synthesis of novel polycyclic and multi-substituted thiophene compounds. Thiophene derivatives $\mathbf{5 a}-\mathbf{c}$ bearing the moiety of 1,4-diketone are ready for further elaboration to numerous heterocyclic compounds, e.g., condensation with hydrazine to form thieno[2,3- $d$ ]pyridazine derivatives $\mathbf{1 7} \mathbf{a}$ and $\mathbf{1 7} \mathbf{c}$ as demonstrated in this study.

\section{Experimental}

\subsection{General}

All reactions requiring anhydrous conditions were conducted in flame-dried apparatus under an atmosphere of nitrogen. Syringes and needles for the transfer of reagents were dried at $100{ }^{\circ} \mathrm{C}$ and allowed to cool in a desiccator over $\mathrm{P}_{2} \mathrm{O}_{5}$ before use. Ethers were distilled from sodium benzophenone ketyl; chlorinated hydrocarbons from $\mathrm{CaH}_{2}$. Reactions were monitored by thin-layer chromatography using pre-coated aluminum plates with a $0.25 \mathrm{~mm}$ layer of silica gel containing a fluorescent indicator (Merck Art. 5544). Column chromatography was carried out on Kieselgel $60(40-63 \mu \mathrm{m})$.

Melting points were recorded using a Yanagimoto micromelting point apparatus and were uncorrected. Chemical 
shifts of ${ }^{1} \mathrm{H}$ and ${ }^{13} \mathrm{C}$ NMR spectra are reported relative to $\mathrm{CHCl}_{3}\left[\delta_{\mathrm{H}} 7.24, \delta_{\mathrm{C}}\right.$ (central line of t) 77.0]. Coupling constants $(J)$ are given in hertz. Distortionless enhancement polarization transfer (DEPT) spectra were taken to determine the types of carbon signals.

4.1.1. Methyl 5-(3,4-dimethoxybenzoyl)-4-[1-hydroxy-1(4-methoxyphenyl)ethyl]-4,5-dihydrothiophene-2-carboxylate (6a). Under an atmosphere of argon, a deep blue $\mathrm{SmI}_{2}$ solution $(0.1 \mathrm{M})$ was prepared by the treatment of $\mathrm{Sm}$ (661 mg, $4.4 \mathrm{mmol})$ with 1,2-diiodoethane (1.01 g, $3.6 \mathrm{mmol})$ in HMPA $(2.8 \mathrm{~mL}, 16 \mathrm{mmol})$ and anhydrous THF $(20 \mathrm{~mL})$ for $1.5 \mathrm{~h}$ at room temperature. To the $\mathrm{SmI}_{2}$ solution (cooled in an ice bath) was added a THF solution (3 mL) of methyl thiophene-2-carboxylate (142 mg, $1 \mathrm{mmol}$ ) and 3,4-dimethoxybenzaldehyde (167 mg, $1.0 \mathrm{mmol})$. The reaction mixture was stirred at $0{ }^{\circ} \mathrm{C}$ for $45 \mathrm{~min}$, and then at room temperature $\left(25^{\circ} \mathrm{C}\right)$ for another $45 \mathrm{~min}$. A solution of 4-methoxyacetophenone $(180 \mathrm{mg}, 1.2 \mathrm{mmol})$ in THF $(2 \mathrm{~mL})$ was added at $0{ }^{\circ} \mathrm{C}$, and the mixture was stirred at $0-25{ }^{\circ} \mathrm{C}$ for additional $10 \mathrm{~h}$. The reaction was quenched by the addition of saturated aqueous $\mathrm{NH}_{4} \mathrm{Cl}$ solution $(0.1 \mathrm{~mL})$. The mixture was passed through a short silica gel column by eluting with EtOAc/hexane (1:1). The filtrate was concentrated, and chromatographed on a silica gel column by eluting with EtOAc/hexane (3:7) to give the desired coupling product $4 \mathbf{a}(354 \mathrm{mg})$ containing two isomers (45:55) as shown by the ${ }^{1} \mathrm{H}$ NMR analysis.

Without further purification, $4 \mathbf{a}(354 \mathrm{mg}, 0.78 \mathrm{mmol})$ in benzene $(10 \mathrm{~mL})$ was stirred with DDQ $(216 \mathrm{mg}, 0.94 \mathrm{mmol})$ at room temperature for $4 \mathrm{~h}$. The reaction mixture was concentrated under reduced pressure, and chromatographed on a silica gel column by elution with EtOAc/hexane (1:2) to afford the corresponding ketone $\mathbf{6 a}(317 \mathrm{mg})$ in $69 \%$ overall yield.

Compound 6a: oil; TLC (EtOAc/hexane, 1:1) $R_{f}=0.30$; IR (neat) 3502, 1709, 1665, 1265, $749 \mathrm{~cm}^{-1} ;{ }^{1} \mathrm{H}$ NMR $\left(\mathrm{CDCl}_{3}, 200 \mathrm{MHz}\right) \delta 7.49(1 \mathrm{H}, \mathrm{d}, J=1.9 \mathrm{~Hz}), 7.45(1 \mathrm{H}, \mathrm{dd}$, $J=8.4,1.9 \mathrm{~Hz}), 7.37(2 \mathrm{H}, \mathrm{d}, J=8.6 \mathrm{~Hz}), 6.85(2 \mathrm{H}, \mathrm{d}$, $J=8.6 \mathrm{~Hz}), 6.84(1 \mathrm{H}, \mathrm{d}, J=8.4 \mathrm{~Hz}), 6.27(1 \mathrm{H}, \mathrm{d}, J=3.0 \mathrm{~Hz})$, $5.36(1 \mathrm{H}, \mathrm{d}, J=7.2 \mathrm{~Hz}), 4.67(1 \mathrm{H}, \mathrm{dd}, J=7.2,3.0 \mathrm{~Hz}), 3.91$ $(3 \mathrm{H}, \mathrm{s}), 3.90(3 \mathrm{H}, \mathrm{s}), 3.77(3 \mathrm{H}, \mathrm{s}), 3.65(3 \mathrm{H}, \mathrm{s}), 2.18(1 \mathrm{H}$, br s, OH), $1.46(3 \mathrm{H}, \mathrm{s}) ;{ }^{13} \mathrm{C} \mathrm{NMR}\left(\mathrm{CDCl}_{3}, 75 \mathrm{MHz}\right)$ $\delta$ 192.5, 161.8, 158.4, 153.6, 149.0, 137.6, 134.6, 133.1, $127.9,126.0(2 \times), 123.1,113.5(2 \times), 110.6,109.9,75.6$, 61.1, 55.9, 55.7, 54.9, 52.1, 50.1, 27.8; MS $m / z$ (rel intensity) 440 (11, $\left.\mathrm{M}^{+}-\mathrm{H}_{2} \mathrm{O}\right), 165$ (100); HRMS calcd for $\mathrm{C}_{24} \mathrm{H}_{26} \mathrm{O}_{7} \mathrm{~S}$ : 458.1399, found: $m / z 458.1400\left(\mathrm{M}^{+}\right)$.

4.1.2. Methyl 4-[1-hydroxy-1-(4-methoxyphenyl)ethyl]5-(4-methoxybenzoyl)-4,5-dihydrothiophene-2-carboxylate (6b). According to the procedure similar to that for 6a, the $\mathrm{SmI}_{2}$-promoted three-component coupling reaction of methyl thiophene-2-carboxylate (142 mg, $1 \mathrm{mmol}), 4-$ methoxybenzaldehyde $(0.12 \mathrm{~mL}, 1.0 \mathrm{mmol})$, and 4-methoxyacetophenone (180 mg, $1.2 \mathrm{mmol})$ afforded $4 \mathbf{b}$ (323 $\mathrm{mg})$. Without further purification, a solution of $\mathbf{4 b}(323 \mathrm{mg}$, $0.75 \mathrm{mmol})$ in $\mathrm{CH}_{2} \mathrm{Cl}_{2}(15 \mathrm{~mL})$ was treated with pyridinium dichromate $(376 \mathrm{mg}, 1.0 \mathrm{mmol})$ at room temperature for $3 \mathrm{~h}$ in the presence of molecular sieves ( $4 \stackrel{\circ}{\mathrm{A}}, 2 \mathrm{~g})$. The mixture was subjected to silica gel column chromatography by eluting with EtOAc/hexane (3:7) to give ketone $6 \mathbf{b}$ (232 $\mathrm{mg}$ ) in $54 \%$ overall yield.

Compound 6b: oil; TLC (EtOAc/hexane, 3:7) $R_{f}=0.17$; IR (neat) 3492, 1708, 666, $1252 \mathrm{~cm}^{-1} ;{ }^{1} \mathrm{H} \mathrm{NMR}\left(\mathrm{CDCl}_{3}\right.$, $200 \mathrm{MHz}) \delta 7.86(2 \mathrm{H}, \mathrm{d}, J=8.9 \mathrm{~Hz}), 7.37(2 \mathrm{H}, \mathrm{d}, J=8.8 \mathrm{~Hz})$, $6.90(2 \mathrm{H}, \mathrm{d}, J=8.9 \mathrm{~Hz}), 6.84(2 \mathrm{H}, \mathrm{d}, J=8.8 \mathrm{~Hz}), 6.28(1 \mathrm{H}, \mathrm{d}$, $J=3.1 \mathrm{~Hz}), 5.34(1 \mathrm{H}, \mathrm{d}, J=7.0 \mathrm{~Hz}), 4.66(1 \mathrm{H}, \mathrm{dd}, J=7.0$, $3.1 \mathrm{~Hz}), 3.82(3 \mathrm{H}, \mathrm{s}), 3.75(3 \mathrm{H}, \mathrm{s}), 3.64(3 \mathrm{H}, \mathrm{s}), 2.90(1 \mathrm{H}$, br s), $1.46(3 \mathrm{H}, \mathrm{s}) ;{ }^{13} \mathrm{C} \mathrm{NMR}\left(\mathrm{CDCl}_{3}, 50 \mathrm{MHz}\right) \delta 192.5$, $163.9,161.9,158.6,137.7,134.6,133.4,131.0(2 \times), 127.9$, $126.1(2 \times), 114.0(2 \times), 113.7(2 \times), 75.9,61.1,55.4,55.1$, 52.3, 50.4, 28.0; MS $m / z$ (rel intensity) $428\left(1, \mathrm{M}^{+}\right), 151$ (100); HRMS calcd for $\mathrm{C}_{23} \mathrm{H}_{24} \mathrm{O}_{6} \mathrm{~S}: 428.1294$, found: $\mathrm{m} / z$ $428.1298\left(\mathrm{M}^{+}\right)$.

4.1.3. Methyl 4-[1-hydroxy-1-(4-methoxyphenyl)ethyl]5-(4-methylbenzoyl)-4,5-dihydrothiophene-2-carboxylate (6c). According to the procedure similar to that for $\mathbf{6 a}$, the $\mathrm{SmI}_{2}$-promoted three-component coupling reaction of methyl thiophene-2-carboxylate $(142 \mathrm{mg}, 1 \mathrm{mmol})$ with 4 methylbenzaldehyde $(0.12 \mathrm{~mL}, 1.0 \mathrm{mmol})$ and 4-methoxyacetophenone (180 mg, $1.2 \mathrm{mmol})$ afforded $4 \mathbf{c}(307 \mathrm{mg})$. Without further purification, a solution of $\mathbf{4 c}(307 \mathrm{mg}$, $0.75 \mathrm{mmol})$ in $\mathrm{CH}_{2} \mathrm{Cl}_{2}(10 \mathrm{~mL})$ was treated with pyridinium dichromate $(420 \mathrm{mg}, 1.12 \mathrm{mmol})$ at room temperature for $1 \mathrm{~h}$ in the presence of molecular sieves $(4 \AA, 1.5 \mathrm{~g})$. The mixture was subjected to silica gel column chromatography by eluting with EtOAc/hexane (1:4) to give ketone $\mathbf{6 c}$ (226 mg) in 54\% overall yield.

Compound 6c: oil; TLC (EtOAc/hexane, 3:7) $R_{f}=0.23$; IR (neat) 3494, 1711, 1671, $1249 \mathrm{~cm}^{-1} ;{ }^{1} \mathrm{H} \mathrm{NMR}\left(\mathrm{CDCl}_{3}\right.$, $200 \mathrm{MHz}) \delta 7.78(2 \mathrm{H}, \mathrm{d}, J=8.2 \mathrm{~Hz}), 7.38(2 \mathrm{H}, \mathrm{dd}, J=8.9$, $2.0 \mathrm{~Hz}), 7.24(2 \mathrm{H}, \mathrm{d}, J=8.2 \mathrm{~Hz}), 6.86(2 \mathrm{H}, \mathrm{dd}, J=8.9$, $2.0 \mathrm{~Hz}), 6.29(1 \mathrm{H}, \mathrm{d}, J=3.1 \mathrm{~Hz}), 5.34(1 \mathrm{H}, \mathrm{d}, J=6.8 \mathrm{~Hz})$, $4.67(1 \mathrm{H}, \mathrm{dd}, J=6.8,3.1 \mathrm{~Hz}), 3.78(3 \mathrm{H}, \mathrm{s}), 3.67(3 \mathrm{H}, \mathrm{s}), 2.39$ $(3 \mathrm{H}, \mathrm{s}), 2.14(1 \mathrm{H}$, br s$), 1.48(3 \mathrm{H}, \mathrm{s}) ;{ }^{13} \mathrm{C} \mathrm{NMR}\left(\mathrm{CDCl}_{3}\right.$, $50 \mathrm{MHz}) \delta 193.4,161.9,158.6,144.6,137.6,134.5,133.4$, $132.4,129.4(2 \times), 128.7(2 \times), 126.1(2 \times), 113.6(2 \times)$, 75.9, 61.0, 55.1, 52.3, 50.6, 27.9, 21.6; MS m/z (rel intensity) $412\left(7, \mathrm{M}^{+}\right), 151$ (100); HRMS calcd for $\mathrm{C}_{23} \mathrm{H}_{24} \mathrm{O}_{5} \mathrm{~S}$ : 412.1344, found: $m / z 412.1340\left(\mathrm{M}^{+}\right)$.

4.1.4. Methyl 5-(3,4-dimethoxybenzoyl)-4-[1-(4-methoxyphenyl)ethenyl]-4,5-dihydrothiophene-2-carboxylate (7). Under an atmosphere of nitrogen, a mixture of alcohol 6a $(25 \mathrm{mg}, 0.054 \mathrm{mmol})$ and $p$-TsOH monohydrate (catalytic amount, ca. $1 \mathrm{mg})$ in benzene $(20 \mathrm{~mL})$ was heated at reflux for $5 \mathrm{~h}$, while the generated water was removed by a Dean-Stark apparatus. The reaction mixture was concentrated under reduced pressure, and chromatographed on a silica gel column by eluting with EtOAc/hexane (1:9) to afford the corresponding alkene 7 (23 mg, 94\% yield) as an oil. TLC (EtOAc/hexane, 3:7) $R_{f}=0.12$; IR (neat) 1710, 1663, 1599, 1262, $748 \mathrm{~cm}^{-1}$; ${ }^{1} \mathrm{H}$ NMR $\left(\mathrm{CDCl}_{3}, 200 \mathrm{MHz}\right) \delta$ $7.46(1 \mathrm{H}, \mathrm{d}, J=1.9 \mathrm{~Hz}), 7.32(2 \mathrm{H}, \mathrm{d}, J=8.7 \mathrm{~Hz}), 7.28(1 \mathrm{H}$, $\mathrm{dd}, J=8.5,1.9 \mathrm{~Hz}), 6.81(2 \mathrm{H}, \mathrm{d}, J=8.7 \mathrm{~Hz}), 6.80(1 \mathrm{H}, \mathrm{d}$, $J=8.5 \mathrm{~Hz}), 6.66(1 \mathrm{H}, \mathrm{d}, J=3.4 \mathrm{~Hz}), 5.44(1 \mathrm{H}, \mathrm{s}), 5.23(1 \mathrm{H}$, $\mathrm{dd}, J=4.3,3.4 \mathrm{~Hz}), 5.15(1 \mathrm{H}, \mathrm{s}), 4.87(1 \mathrm{H}, \mathrm{d}, J=4.3 \mathrm{~Hz})$, $3.89(6 \mathrm{H}, \mathrm{s}), 3.75(3 \mathrm{H}, \mathrm{s}), 3.74(3 \mathrm{H}, \mathrm{s}) ;{ }^{13} \mathrm{C} \mathrm{NMR}\left(\mathrm{CDCl}_{3}\right.$, $50 \mathrm{MHz}) \delta 191.9,162.1,159.5,153.7,149.2,145.5,136.5$, $133.0,131.5,127.8,127.5(2 \times), 123.0,114.0(2 \times), 113.2$, 
110.9, 110.0, 56.1, 56.0, 55.2, 54.6, 52.4, 52.3; MS m/z (rel intensity) $440\left(58, \mathrm{M}^{+}\right), 165$ (100); HRMS calcd for $\mathrm{C}_{24} \mathrm{H}_{24} \mathrm{O}_{6} \mathrm{~S}: 440.1294$, found: $m / z 440.1289\left(\mathrm{M}^{+}\right)$.

4.1.5. Methyl 5-(3,4-dimethoxybenzoyl)-4-[1-(4-methoxyphenyl)ethenyl] thiophene-2-carboxylate (8). A solution of alkene $7(150 \mathrm{mg}, 0.34 \mathrm{mmol})$ in degassed anhydrous acetonitrile $(7 \mathrm{~mL})$ was stirred with $\mathrm{Pd}(\mathrm{OAc})_{2}(153 \mathrm{mg}$, $0.68 \mathrm{mmol})$ and $\mathrm{K}_{2} \mathrm{CO}_{3}(142 \mathrm{mg}, 1.02 \mathrm{mmol})$ at room temperature for $12 \mathrm{~h}$. The reaction mixture was concentrated under reduced pressure, and chromatographed on a silica gel column by eluting with EtOAc/hexane (1:9) to give compound 8 (136 $\mathrm{mg}, 91 \%$ yield) as an oil. TLC $\left(\mathrm{CH}_{2} \mathrm{Cl}_{2}\right)$ $R_{f}=0.35$; IR (neat) $1718,1637,1594,1511,1268 \mathrm{~cm}^{-1}$; ${ }^{1} \mathrm{H}$ NMR $\left(\mathrm{CDCl}_{3}, 300 \mathrm{MHz}\right) \delta 7.74(1 \mathrm{H}, \mathrm{s}), 7.14(1 \mathrm{H}, \mathrm{dd}$, $J=8.2,2.0 \mathrm{~Hz}), 6.93(1 \mathrm{H}, \mathrm{d}, J=2.0 \mathrm{~Hz}), 6.85(2 \mathrm{H}, \mathrm{dd}, J=$ $8.8,2.0 \mathrm{~Hz}), 6.65(1 \mathrm{H}, \mathrm{d}, J=8.2 \mathrm{~Hz}), 6.60(2 \mathrm{H}, \mathrm{dd}, J=8.8$, $2.0 \mathrm{~Hz}), 5.29(1 \mathrm{H}, \mathrm{s}), 5.20(1 \mathrm{H}, \mathrm{s}), 3.86(3 \mathrm{H}, \mathrm{s}), 3.84(3 \mathrm{H}$, s), $3.71(3 \mathrm{H}, \mathrm{s}), 3.69(3 \mathrm{H}, \mathrm{s}) ;{ }^{13} \mathrm{C} \mathrm{NMR}\left(\mathrm{CDCl}_{3}, 75 \mathrm{MHz}\right)$ $\delta 188.1,161.9,159.1,153.2,148.5,144.8,143.6,142.9$, $135.4,134.9,132.9,130.1,127.9(2 \times), 124.4,115.3,113.4$ $(2 \times), 110.4,109.4,55.9,55.6,55.0,52.4 ; \mathrm{MS} m / z$ (rel intensity) $438\left(31, \mathrm{M}^{+}\right), 55(100)$; HRMS calcd for $\mathrm{C}_{24} \mathrm{H}_{22} \mathrm{O}_{6} \mathrm{~S}$ : 438.1137, found: $m / z 438.1140\left(\mathrm{M}^{+}\right)$.

4.1.6. Methyl 6,7-dimethoxy-4-(4-methoxyphenyl)-4methyl-9-oxo-4,9-dihydronaphtho[2,3- $b]$ thiophene-2carboxylate (9). Compound $8(55 \mathrm{mg}, 0.125 \mathrm{mmol})$ and catalytic amount of $p$-TsOH monohydrate in $\mathrm{CH}_{2} \mathrm{Cl}_{2}$ solution $(15 \mathrm{~mL})$ were stirred at room temperature for $2 \mathrm{~h}$. The reaction mixture was filtered, concentrated under reduced pressure, and chromatographed on a silica gel column by eluting with EtOAc/hexane (1:9) to give compound 9 (53 mg, 97\% yield) as an oil; TLC (EtOAc/hexane, 3:7) $R_{f}=0.23$; IR (neat) $1719,645,1279 \mathrm{~cm}^{-1} ;{ }^{1} \mathrm{H}$ NMR $\left(\mathrm{CDCl}_{3}, 200 \mathrm{MHz}\right) \delta 7.73(1 \mathrm{H}, \mathrm{s}), 7.39(1 \mathrm{H}, \mathrm{s}), 7.07(2 \mathrm{H}$, dd, $J=8.6,2.1 \mathrm{~Hz}), 6.77(2 \mathrm{H}, \mathrm{dd}, J=8.6,2.1 \mathrm{~Hz}), 6.47$ $(1 \mathrm{H}, \mathrm{s}), 3.95(3 \mathrm{H}, \mathrm{s}), 3.83(3 \mathrm{H}, \mathrm{s}), 3.74(3 \mathrm{H}, \mathrm{s}), 3.73(3 \mathrm{H}$, s), $1.94(3 \mathrm{H}, \mathrm{s}) ;{ }^{13} \mathrm{C} \mathrm{NMR}\left(\mathrm{CDCl}_{3}, 75 \mathrm{MHz}\right) \delta 178.0,162.2$, $158.4,157.3,153.7,148.3,146.2,139.3,139.1,136.2,133.3$, $128.1(2 \times), 123.9,114.0(2 \times), 110.2,107.3,56.0,55.9$, 55.1, 52.5, 46.2, 30.4; MS $\mathrm{m} / \mathrm{z}$ (rel intensity) $438\left(75, \mathrm{M}^{+}\right)$, 423 (100); HRMS calcd for $\mathrm{C}_{24} \mathrm{H}_{22} \mathrm{O}_{6} \mathrm{~S}$ : 438.1137, found: $\mathrm{m} / z, 438.1142\left(\mathrm{M}^{+}\right)$.

4.1.7. Methyl 4-[1-hydroxy-1-(4-methoxyphenyl)ethyl]5-(1-methylindole-2-carbonyl)-4,5-dihydrothiophene-2carboxylate (11). According to the procedure similar to that for $\mathbf{4 a}$, the $\mathrm{SmI}_{2}$-promoted three-component coupling reaction of methyl thiophene-2-carboxylate $(142 \mathrm{mg}, 1 \mathrm{mmol})$ with $N$-methylindole-2-carboxaldehyde $(159 \mathrm{mg}, 1 \mathrm{mmol})$ and 4-methoxyacetophenone (180 mg, $1.2 \mathrm{mmol})$ afforded $10(349 \mathrm{mg}$ ) containing two isomers $(65: 35)$ as shown by the ${ }^{1} \mathrm{H}$ NMR analysis. Without further purification, 10 (349 mg, $0.77 \mathrm{mmol}$ ) was treated with DDQ (216 mg, $0.94 \mathrm{mmol}$ ) by a procedure similar to that for $\mathbf{6 a}$ to give ketone $11(306 \mathrm{mg})$ in 65\% overall yield. Compound 11: oil; TLC (EtOAc/hexane, 3:7) $R_{f}=0.23$; IR (neat) 3502, 1717, $1661,1613,1251,753 \mathrm{~cm}^{-1}$; ${ }^{1} \mathrm{H}$ NMR $\left(\mathrm{CDCl}_{3}, 300 \mathrm{MHz}\right)$ $\delta 7.66(1 \mathrm{H}, \mathrm{d}, J=8.0 \mathrm{~Hz}), 7.41(2 \mathrm{H}, \mathrm{d}, J=8.7 \mathrm{~Hz}), 7.38$ $7.33(2 \mathrm{H}, \mathrm{m}), 7.20(1 \mathrm{H}, \mathrm{s}), 7.13(1 \mathrm{H}, \mathrm{td}, J=8.0,2.0 \mathrm{~Hz})$, $6.86(2 \mathrm{H}, \mathrm{d}, J=8.7 \mathrm{~Hz}), 6.34(1 \mathrm{H}, \mathrm{d}, J=3.0 \mathrm{~Hz}), 5.42(1 \mathrm{H}$, d, $J=7.2 \mathrm{~Hz}), 4.64(1 \mathrm{H}, \mathrm{dd}, J=7.2,3.0 \mathrm{~Hz}), 4.03(3 \mathrm{H}, \mathrm{s})$,
$3.74(3 \mathrm{H}, \mathrm{s}), 3.67(3 \mathrm{H}, \mathrm{s}), 2.40(1 \mathrm{H}, \mathrm{br} \mathrm{s}, \mathrm{OH}), 1.54(3 \mathrm{H}$, $\mathrm{s}) ;{ }^{13} \mathrm{C} \mathrm{NMR}\left(\mathrm{CDCl}_{3}, 75 \mathrm{MHz}\right) \delta 187.6,162.0,158.6$, 140.6, 137.6, 134.3, 133.8, 133.1, 126.4, $126.1(2 \times), 125.6$, $123.1,120.9,113.7(2 \times), 112.1,110.3,75.9,61.0,55.1$, 52.3, 52.0, 32.1, 27.7; FABMS $m / z 452.1\left(\mathrm{M}^{+}-\mathrm{H}_{2} \mathrm{O}+\mathrm{H}\right)$; HRMS calcd for $\mathrm{C}_{25} \mathrm{H}_{25} \mathrm{NO}_{5} \mathrm{~S}: 451.1453$, found: $\mathrm{m} / \mathrm{z}$ $451.1463\left(\mathrm{M}^{+}\right)$.

4.1.8. Methyl 4-[1-(4-methoxyphenyl)ethenyl]-5-(1methylindole-2-carbonyl)-4,5-dihydrothiophene-2-carboxylate (12). By a procedure similar to that for 7 , treatment of $11(95 \mathrm{mg}, 0.21 \mathrm{mmol})$ with catalytic amount of $p-\mathrm{TsOH}$ monohydrate (ca. $3 \mathrm{mg}$ ) in refluxing benzene for $5 \mathrm{~h}$ gave the dehydration product $\mathbf{1 2}(87 \mathrm{mg}, 95 \%)$ as an oil; TLC (EtOAc/hexane, 3:7) $R_{f}=0.13$; IR (neat) 1720, 1660, 1607, $1511,1250 \mathrm{~cm}^{-1}$; ${ }^{1} \mathrm{H}$ NMR $\left(\mathrm{CDCl}_{3}, 300 \mathrm{MHz}\right) \delta 7.59$ $(1 \mathrm{H}, \mathrm{d}, J=8.0 \mathrm{~Hz}), 7.36-7.32(4 \mathrm{H}, \mathrm{m}), 7.14-7.08(1 \mathrm{H}, \mathrm{m})$, $6.97(1 \mathrm{H}, \mathrm{s}), 6.83(2 \mathrm{H}, \mathrm{d}, J=8.7 \mathrm{~Hz}), 6.69(1 \mathrm{H}, \mathrm{d}$, $J=3.5 \mathrm{~Hz}), 5.47(1 \mathrm{H}, \mathrm{s}), 5.20(1 \mathrm{H}, \mathrm{dd}, J=4.6,3.5 \mathrm{~Hz})$, $5.19(1 \mathrm{H}, \mathrm{s}), 4.98(1 \mathrm{H}, \mathrm{d}, J=4.6 \mathrm{~Hz}), 4.06(3 \mathrm{H}, \mathrm{s}), 3.77$ $(3 \mathrm{H}, \mathrm{s}), 3.74(3 \mathrm{H}, \mathrm{s}) ;{ }^{13} \mathrm{C} \mathrm{NMR}\left(\mathrm{CDCl}_{3}, 75 \mathrm{MHz}\right) \delta 187.3$, $162.1,159.4,145.6,140.5,136.1,133.4,132.8,131.5$, $127.5(2 \times), 126.3,125.6,122.9,120.9,113.9(2 \times), 113.2$, $111.5,110.3,56.2,55.1,52.4,52.3,32.1 ; \mathrm{MS} \mathrm{m} / \mathrm{z}$ (rel intensity) $433\left(57, \mathrm{M}^{+}\right), 374(13), 275(100), 158(88), 133$ (32); HRMS calcd for $\mathrm{C}_{25} \mathrm{H}_{23} \mathrm{NO}_{4} \mathrm{~S}$ : 433.1348, found: $\mathrm{m} / \mathrm{z}$ $433.1346\left(\mathrm{M}^{+}\right)$.

4.1.9. Methyl 4-[1-(4-methoxyphenyl)ethenyl]-5(1-methylindole-2-carbonyl)thiophene-2-carboxylate (13). By a procedure similar to that for 8 , alkene $12(70 \mathrm{mg}$, $0.16 \mathrm{mmol}$ ) was treated with $\mathrm{Pd}(\mathrm{OAc})_{2}(70 \mathrm{mg}, 0.31 \mathrm{mmol})$ and $\mathrm{K}_{2} \mathrm{CO}_{3}(130 \mathrm{mg}, 0.94 \mathrm{mmol})$ at room temperature for $12 \mathrm{~h}$ to give compound $\mathbf{1 3}(65 \mathrm{mg}, 93 \%)$ as an oil. TLC (EtOAc/hexane, 1:9) $R_{f}=0.13$; IR (neat) 1719, 1626, $1510,1247 \mathrm{~cm}^{-1} ;{ }^{1} \mathrm{H}$ NMR $\left(\mathrm{CDCl}_{3}, 300 \mathrm{MHz}\right) \delta 7.83$ $(1 \mathrm{H}, \mathrm{s}), 7.59(1 \mathrm{H}, \mathrm{d}, J=8.0 \mathrm{~Hz}), 7.35(1 \mathrm{H}, \mathrm{t}, J=8.0 \mathrm{~Hz})$, $7.24(1 \mathrm{H}, \mathrm{d}, J=8.0 \mathrm{~Hz}), 7.11(1 \mathrm{H}, \mathrm{t}, J=8.0 \mathrm{~Hz}), 6.99(1 \mathrm{H}$, s), $6.82(2 \mathrm{H}, \mathrm{d}, J=8.5 \mathrm{~Hz}), 6.59(2 \mathrm{H}, \mathrm{d}, J=8.5 \mathrm{~Hz}), 5.34$ $(1 \mathrm{H}, \mathrm{s}), 5.33(1 \mathrm{H}, \mathrm{s}), 3.92(3 \mathrm{H}, \mathrm{s}), 3.70(3 \mathrm{H}, \mathrm{s}), 3.57(3 \mathrm{H}$, $\mathrm{s}) ;{ }^{13} \mathrm{C}$ NMR $\left(75 \mathrm{MHz}, \mathrm{CDCl}_{3}\right) \delta 180.5,162.2,159.2$, $145.6,143.9,143.5,140.3,135.7,135.2,135.0,133.5$, $128.0(2 \times), 126.2,125.7,123.0,120.7,115.1,114.9,113.3$ $(2 \times), 110.1,55.2,52.5,31.1$; FABMS $\mathrm{m} / \mathrm{z} 431\left(\mathrm{M}^{+}\right)$; HRMS calcd for $\mathrm{C}_{25} \mathrm{H}_{21} \mathrm{NO}_{4} \mathrm{~S}:$ 431.1191, found: $\mathrm{m} / \mathrm{z}$ $431.1183\left(\mathrm{M}^{+}\right)$.

4.1.10. Methyl 4,9-dimethyl-4-(4-methoxyphenyl)-10oxo-4,10-dihydrocarbazolo[2,3-b] thiophene-2-carboxylate (14). By a procedure similar to that for 9 , treatment of $\mathbf{1 3}$ (50 mg, $0.12 \mathrm{mmol}$ ) with a catalytic amount of $p$-TsOH monohydrate in $\mathrm{CH}_{2} \mathrm{Cl}_{2}$ solution at room temperature for $2 \mathrm{~h}$ afforded the acid-catalyzed cyclization product 14 (49 mg, 98\%) as colorless solid, $\mathrm{mp} 113-114^{\circ} \mathrm{C}$. TLC (EtOAc/hexane, 1:9) $R_{f}=0.13$; IR (KBr) 1716, 1637, 1510, $1247 \mathrm{~cm}^{-1} ;{ }^{1} \mathrm{H}$ NMR $\left(\mathrm{CDCl}_{3}, 200 \mathrm{MHz}\right) \delta 7.53(1 \mathrm{H}, \mathrm{s})$, 7.38-7.30 $(2 \mathrm{H}, \mathrm{m}), 7.23-7.16(3 \mathrm{H}, \mathrm{m}), 6.97(1 \mathrm{H}, \mathrm{m}), 6.77$ $(2 \mathrm{H}, \mathrm{dd}, J=8.8,2.1 \mathrm{~Hz}), 4.26(3 \mathrm{H}, \mathrm{s}), 3.85(3 \mathrm{H}, \mathrm{s}), 3.73$ $(3 \mathrm{H}, \mathrm{s}), 2.08(3 \mathrm{H}, \mathrm{s}) ;{ }^{13} \mathrm{C} \mathrm{NMR}\left(\mathrm{CDCl}_{3}, 75 \mathrm{MHz}\right) \delta 173.7$, $162.3,158.4,158.0,141.8,140.5,138.4,135.6,134.9$, $132.7,128.8,127.8(2 \times), 126.5,123.2,122.2,120.4,114.1$ (2×), 110.5, 55.1, 52.5, 44.4, 31.6, 28.1; FABMS m/z 431 $\left(\mathrm{M}^{+}\right)$; HRMS calcd for $\mathrm{C}_{25} \mathrm{H}_{21} \mathrm{NO}_{4} \mathrm{~S}: 431.1191$, found: $\mathrm{m} / \mathrm{z}$ 
431.1192 $\left(\mathrm{M}^{+}\right)$. Anal. Calcd for $\mathrm{C}_{25} \mathrm{H}_{21} \mathrm{NO}_{4} \mathrm{~S}: \mathrm{C}, 69.59 ; \mathrm{H}$, 4.91; N, 3.25. Found: C, 69.42; H, 4.98; N, 3.14.

\subsubsection{Methyl 5-(3,4-dimethoxybenzoyl)-4-[1-(4-meth-} oxyphenyl)ethylidene]-5H-thiophene-2-carboxylate (15a). Under an atmosphere of nitrogen, a mixture of $\mathbf{6 a}$ (263 mg, $0.59 \mathrm{mmol}$ ) and $p$-TsOH monohydrate $(57 \mathrm{mg}$, $0.30 \mathrm{mmol})$ in benzene $(20 \mathrm{~mL})$ was heated at reflux for $1 \mathrm{~h}$. The mixture was then subjected to silica gel column chromatography by eluting with EtOAc/hexane (1:4) to give alkene 15a (204 mg, 79\% yield) as a solid; $\mathrm{mp} 156-158{ }^{\circ} \mathrm{C}$; TLC (EtOAc/hexane, 1:4) $R_{f}=0.14$; IR (KBr) 1701, 1669, $1245,751 \mathrm{~cm}^{-1} ;{ }^{1} \mathrm{H} \mathrm{NMR}\left(\mathrm{CDCl}_{3}, 300 \mathrm{MHz}\right) \delta 7.57(1 \mathrm{H}$, s), $7.56(1 \mathrm{H}, \mathrm{d}, J=8.1 \mathrm{~Hz}), 7.21(2 \mathrm{H}, \mathrm{d}, J=8.5 \mathrm{~Hz}), 7.04$ $(1 \mathrm{H}, \mathrm{s}), 6.91(1 \mathrm{H}, \mathrm{d}, J=8.1 \mathrm{~Hz}), 6.88(2 \mathrm{H}, \mathrm{d}, J=8.5 \mathrm{~Hz})$, $5.86(1 \mathrm{H}, \mathrm{s}), 3.95(3 \mathrm{H}, \mathrm{s}), 3.92(3 \mathrm{H}, \mathrm{s}), 3.81(3 \mathrm{H}, \mathrm{s}), 3.70$ $(3 \mathrm{H}, \mathrm{s}), 1.90(3 \mathrm{H}, \mathrm{s}) ;{ }^{13} \mathrm{C} \mathrm{NMR}\left(\mathrm{CDCl}_{3}, 75 \mathrm{MHz}\right) \delta 190.3$, $162.9,159.2,153.8,149.3,141.6,138.3,134.9,134.4$, 133.7, 129.2 (2×), 128.0, 122.8, $113.7(2 \times), 110.9,110.1$, $56.1,55.9,55.3,54.1,52.3,22.6$; MS $\mathrm{m} / \mathrm{z}$ (rel intensity) $440\left(55, \mathrm{M}^{+}\right), 165(100)$; HRMS calcd for $\mathrm{C}_{24} \mathrm{H}_{24} \mathrm{O}_{6} \mathrm{~S}$ : 440.1293, found: $\mathrm{m} / \mathrm{z} 440.1294\left(\mathrm{M}^{+}\right)$. Anal. Calcd for $\mathrm{C}_{24} \mathrm{H}_{24} \mathrm{O}_{6} \mathrm{~S}: \mathrm{C}, 65.44 ; \mathrm{H}, 5.49$. Found: $\mathrm{C}, 65.68 ; \mathrm{H}, 5.42$.

4.1.12. Methyl 4-acetyl-5-(3,4-dimethoxybenzoyl)thiophene-2-carboxylate (5a). A mixture of $\mathbf{1 5 a}(30 \mathrm{mg}$, $0.07 \mathrm{mmol})$ and stoichiometric amount of $p$-TsOH monohydrate $(19 \mathrm{mg}, 0.1 \mathrm{mmol})$ in $\mathrm{CHCl}_{3}(10 \mathrm{~mL})$ was placed in a round-bottomed flask without capping. The mixture was stirred in air for $2.5 \mathrm{~h}$ at room temperature, and then subjected to a short silica gel column to remove $p-\mathrm{TsOH}$. The crude product eluted by EtOAc/hexane (1:1) was concentrated, and purified by chromatography by eluting with EtOAc/hexane (1:4) to give 4-methoxyphenol (16, $8.1 \mathrm{mg}$, 95\%) and diacylthiophene $\mathbf{5 a}(23 \mathrm{mg}, 95 \%)$.

Compound 5a: solid; mp 125-127 ${ }^{\circ} \mathrm{C}$; TLC (EtOAc/hexane, $1: 4) R_{f}=0.07$; IR (KBr) 1716, 1678, 1650, 1267, $754 \mathrm{~cm}^{-1}$; ${ }^{1} \mathrm{H}$ NMR $\left(\mathrm{CDCl}_{3}, 200 \mathrm{MHz}\right) \delta 8.03(1 \mathrm{H}, \mathrm{s}), 7.52(1 \mathrm{H}, \mathrm{d}$, $J=2.0 \mathrm{~Hz}), 7.21(1 \mathrm{H}, \mathrm{dd}, J=8.4,2.0 \mathrm{~Hz}), 6.78(1 \mathrm{H}, \mathrm{d}$, $J=8.4 \mathrm{~Hz}), 3.90(6 \mathrm{H}, \mathrm{s}), 3.89(3 \mathrm{H}, \mathrm{s}), 2.38(3 \mathrm{H}, \mathrm{s}) ;{ }^{13} \mathrm{C}$ NMR $\left(\mathrm{CDCl}_{3}, 50 \mathrm{MHz}\right) \delta 192.3,187.7,161.4,154.3,150.0$, 149.3, 141.4, 134.3, 132.8, 129.7, 125.4, 110.5, 109.9, 56.1, 56.0, 52.7, 28.8; MS m/z (rel intensity) $348\left(100, \mathrm{M}^{+}\right)$; HRMS calcd for $\mathrm{C}_{17} \mathrm{H}_{16} \mathrm{O}_{6} \mathrm{~S}: 348.0667$, found: $\mathrm{m} / \mathrm{z}$ $348.0670\left(\mathrm{M}^{+}\right)$. Anal. Calcd for $\mathrm{C}_{17} \mathrm{H}_{16} \mathrm{O}_{6} \mathrm{~S}: \mathrm{C}, 58.61 ; \mathrm{H}$, 4.63. Found: C, 58.72; H, 4.56 .

4.1.13. Methyl 4-acetyl-5-(4-methoxybenzoyl)thiophene2-carboxylate $(\mathbf{5 b})$. By a procedure similar to that for $\mathbf{1 5 a}$, alcohol 6b $(182 \mathrm{mg}, 0.42 \mathrm{mmol})$ was first treated with 0.5 equiv of $p$-TsOH $(40 \mathrm{mg}, 0.21 \mathrm{mmol})$ in refluxing benzene under an atmosphere of nitrogen to give a crude product of alkene 15b $(148 \mathrm{mg})$. Without further purification, the crude product was stirred with excess amount of $p$-TsOH (100 mg, $0.53 \mathrm{mmol}$ ) in $\mathrm{CHCl}_{3}$ for $2 \mathrm{~h}$ under an atmosphere of air to give $\mathbf{5 b}(113 \mathrm{mg})$ in $85 \%$ overall yield.

Compound 5b: oil; TLC (EtOAc/hexane, 1:4) $R_{f}=0.09$; IR (neat) $1713,1678,1649,1253 \mathrm{~cm}^{-1} ;{ }^{1} \mathrm{H}$ NMR $\left(\mathrm{CDCl}_{3}\right.$, $200 \mathrm{MHz}) \delta 8.03(1 \mathrm{H}, \mathrm{s}), 7.45(2 \mathrm{H}, \mathrm{dd}, J=8.9,2.0 \mathrm{~Hz})$, $6.88(2 \mathrm{H}, \mathrm{dd}, J=8.9,2.0 \mathrm{~Hz}), 3.89(3 \mathrm{H}, \mathrm{s}), 3.82(3 \mathrm{H}, \mathrm{s})$, $2.38(3 \mathrm{H}, \mathrm{s}) ;{ }^{13} \mathrm{C} \mathrm{NMR}\left(\mathrm{CDCl}_{3}, 50 \mathrm{MHz}\right) \delta 192.2,187.7$,
$164.3,161.4,150.2,141.2,134.2,132.9,131.9(2 \times)$, 129.4, 114.0 (2×) $55.5,52.6,28.8$; MS $\mathrm{m} / \mathrm{z}$ (rel intensity) $318\left(54, \mathrm{M}^{+}\right), 135(100)$; HRMS calcd for $\mathrm{C}_{16} \mathrm{H}_{14} \mathrm{O}_{5} \mathrm{~S}$ : 318.0562, found: $m / z 318.0564\left(\mathrm{M}^{+}\right)$.

4.1.14. 6-(2,3-Diaza-4-methyl-1-oxo-3-penten-1-yl)-1methyl-4-(3,4-dimethoxyphenyl)thieno[ $2,3-d]$ pyridazine (17a). A mixture of 5a $(20 \mathrm{mg}, 0.06 \mathrm{mmol})$ and excess amount of hydrazine monohydrate $(9 \mathrm{mg}, 0.18 \mathrm{mmol})$ in EtOH $(15 \mathrm{~mL})$ was stirred at room temperature for $10 \mathrm{~min}$. The mixture was concentrated, acetone was added $(20 \mathrm{~mL})$, and concentrated again under reduced pressure. This procedure was repeated twice to give the desired product 17a $(23 \mathrm{mg})$ in $99 \%$ yield. Solid; mp $209-211^{\circ} \mathrm{C}$; TLC $\left(\mathrm{MeOH} / \mathrm{CH}_{2} \mathrm{Cl}_{2}, 1: 19\right) R_{f}=0.13$; IR (KBr) 3423, 1642, $1415,1232 \mathrm{~cm}^{-1} ;{ }^{1} \mathrm{H} \mathrm{NMR}\left(\mathrm{CDCl}_{3}, 200 \mathrm{MHz}\right) \delta 9.69(1 \mathrm{H}$, br s), $8.53(1 \mathrm{H}, \mathrm{s}), 7.71(1 \mathrm{H}, \mathrm{s}), 7.69(1 \mathrm{H}, \mathrm{d}, J=8.1 \mathrm{~Hz}), 7.00$ $(1 \mathrm{H}, \mathrm{d}, J=8.1 \mathrm{~Hz}), 3.59(3 \mathrm{H}, \mathrm{s}), 3.94(3 \mathrm{H}, \mathrm{s}), 2.99(3 \mathrm{H}, \mathrm{s})$, $2.13(3 \mathrm{H}, \mathrm{s}), 2.03(3 \mathrm{H}, \mathrm{s}) ;{ }^{13} \mathrm{C} \mathrm{NMR}\left(\mathrm{CDCl}_{3}, 75 \mathrm{MHz}\right)$ $\delta$ 161.5, 154.3, 153.9, 152.3, 150.8, 149.3, 141.0, 139.8, 134.9, 129.8, 128.8, 121.1, 111.3, 110.9, $55.9(2 \times), 25.1$, 19.9, 16.2; MS $m / z$ (rel intensity) $384\left(70, \mathrm{M}^{+}\right), 285(100)$; HRMS calcd for $\mathrm{C}_{19} \mathrm{H}_{20} \mathrm{~N}_{4} \mathrm{O}_{3} \mathrm{~S}$ : 384.1256, found: $\mathrm{m} / \mathrm{z}$ $384.1259\left(\mathrm{M}^{+}\right)$. Anal. Calcd for $\mathrm{C}_{19} \mathrm{H}_{20} \mathrm{~N}_{4} \mathrm{O}_{3} \mathrm{~S}$ : C, 59.36; H, 5.24; N, 14.57. Found: C, 59.46; H, 5.12; N, 14.62.

4.1.15. 6-(2,3-Diaza-4-methyl-1-oxo-3-penten-1-yl)-1methyl-4-(4-methylphenyl)thieno $[2,3-d]$ pyridazine (17c). By a procedure similar to that for $5 \mathbf{b}$, compound $\mathbf{6 c}$ (200 mg, $0.51 \mathrm{mmol}$ ) was first treated with 0.5 equiv of $p$-TsOH in refluxing benzene under an atmosphere of nitrogen to give a crude product $15 \mathrm{c}$. The crude product was subsequently stirred with stoichiometric amount of $p$-TsOH in $\mathrm{CHCl}_{3}$ for $2.5 \mathrm{~h}$ under an atmosphere of air to give a mixture of 4-methoxyphenol and $\mathbf{5 c}$, which were inseparable by silica gel column chromatography. By a procedure similar to that for 17a, the mixture was treated with excess amounts of hydrazine and acetone to afford a crude product, which was purified on a silica gel column by eluting with $\mathrm{MeOH} /$ $\mathrm{CH}_{2} \mathrm{Cl}_{2}(1: 19)$ to give $17 \mathrm{c}(168 \mathrm{mg})$ in $97 \%$ overall yield. Solid; mp 262-264 ${ }^{\circ} \mathrm{C}$; TLC $\left(\mathrm{MeOH} / \mathrm{CH}_{2} \mathrm{Cl}_{2}, 1: 19\right)$ $R_{f}=0.17$; IR (KBr) 3447, 1648, 1390, $1259 \mathrm{~cm}^{-1} ;{ }^{1} \mathrm{H}$ NMR $\left(\mathrm{CDCl}_{3} / \mathrm{CD}_{3} \mathrm{OD}=4: 1,200 \mathrm{MHz}\right) \delta 8.39(1 \mathrm{H}, \mathrm{s}), 7.80$ $(2 \mathrm{H}, J=8.0 \mathrm{~Hz}), 7.23(2 \mathrm{H}, \mathrm{d}, J=8.0 \mathrm{~Hz}), 2.83(3 \mathrm{H}, \mathrm{s}), 2.32$ $(3 \mathrm{H}, \mathrm{s}), 1.98(3 \mathrm{H}, \mathrm{s}), 1.87(3 \mathrm{H}, \mathrm{s}) ;{ }^{13} \mathrm{C} \mathrm{NMR}\left(\mathrm{CDCl}_{3} /\right.$ $\left.\left.\mathrm{CD}_{3} \mathrm{OD}=3: 1\right), 75 \mathrm{MHz}\right) \delta 161.5,154.8,154.1,152.7$, 141.3, 140.4, 140.0, 132.9, 134.7, 129.4 (2×), 129.2, 127.9 (2×), 24.6, 21.0, 19.3, 16.3; MS $\mathrm{m} / \mathrm{z}$ (rel intensity) 338 $\left(42, \mathrm{M}^{+}\right), 239$ (100); HRMS calcd for $\mathrm{C}_{18} \mathrm{H}_{18} \mathrm{~N}_{4} \mathrm{OS}$ : 338.1202, found: $\mathrm{m} / \mathrm{z} 338.1199\left(\mathrm{M}^{+}\right)$. Anal. Calcd for $\mathrm{C}_{18} \mathrm{H}_{18} \mathrm{~N}_{4} \mathrm{OS}$ : C, 63.88; H, 5.36; N, 16.56. Found: C, 63.82; H, 5.40; N, 16.42 .

\section{Acknowledgements}

We thank the National Science Council for financial support.

\section{Supplementary data}

Supplementary data associated with this article can be found in the online version, at doi:10.1016/j.tet.2006.11.080. 


\section{References and notes}

1. (a) Roncali, J. Chem. Rev. 1992, 92, 711; (b) Roncali, J. Chem. Rev. 1997, 97, 173; Miller, L. L.; Mann, K. R. Acc. Chem. Res. 1996, 29, 417; (c) Larsen, J.; Bechgaard, K. Acta Chem. Scand. 1996, 50, 71; (d) Müller, M.; Mauermann-Dull, H.; Wagner, M.; Enkelmann, V.; Müllen, K. Angew. Chem., Int. Ed. Engl. 1995, 34, 1583; (e) Tsuji, K.; Nakamura, K.; Ogino, T.; Konishi, N.; Tojo, T.; Ochi, T.; Seki, N.; Matsuo, M. Chem. Pharm. Bull. 1998, 46, 279; (f) Becker, F. F.; Mukhopadhyay, C.; Hackfeld, L.; Banik, I.; Banik, B. K. Bioorg. Med. Chem. 2000, 8, 2693.

2. For reviews on the synthetic methods of thiophene derivatives, see: (a) Campaigne, E. Comprehensive Heterocyclic Chemistry; Katritzky, A. R., Rees, C. W., Eds.; Elsevier: Oxford, 1984; Vol. 4, pp 863-934; (b) Nakayama, J. Comprehensive Heterocyclic Chemistry II; Katritzky, A. R., Rees, C. W., Scriven, E. F. V., Eds.; Elsevier: Oxford, 1996; Vol. 2, pp 607-677.

3. (a) Kozmík, V.; Paleèek, J. Collect. Czech. Chem. Commun. 1992, 57, 1483; (b) Sniekus, V. Chem. Rev. 1990, 90, 879; (c) MacDowell, D. W. H.; Ballas, F. L. J. Org. Chem. 1977, 42, 3717; (d) Beleńkii, L. I.; Gromova, G. P.; Kolotaev, A. V.; Krayushkin, M. M. Chem. Heterocycl. Compd. (Engl. Transl.) 2000, 36, 256; (e) Pirson, P.; Schonne, A.; Christiaens, L. Bull. Soc. Chim. Belg. 1970, 79, 575.

4. (a) Yang, S.-M.; Fang, J.-M. Tetrahedron Lett. 1997, 38, 1589; (b) Yang, S.-M.; Nandy, S. K.; Selvakumar, A. R.; Fang, J.-M. Org. Lett. 2000, 2, 3719; (c) Shie, J.-J.; Yang,
S.-M.; Chen, C.-T.; Fang, J.-M. Org. Lett. 2002, 4, 1099; (d) Yang, S.-M.; Shie, J.-J.; Fang, J.-M.; Nandy, S. K.; Chang, H.-Y.; Lu, S.-H.; Wang, G. J. Org. Chem. 2002, 67, 5208.

5. Babu, G.; Yu, H.-M.; Yang, S.-M.; Fang, J.-M. Bioorg. Med. Chem. Lett. 2004, 14, 1129.

6. For biological role of endothelins, see: (a) Yanagisawa, M.; Kurihara, H.; Kimura, S.; Tomobe, Y.; Kobayashi, M.; Mitsui, Y.; Yazaki, Y.; Goto, K.; Masaki, T. Nature 1988, 332, 311; (b) Doherty, A. M. J. Med. Chem. 1992, 35, 1493; (c) Schiffrin, E. L.; Touyz, R. M. J. Cardiovasc. Pharmacol. 1998, 32, 2; (d) For review on endothelin antagonists, see: Elliott, J. D.; Lago, M. A.; Peishoff, C. E. Endothelin Receptors: from the Gene to the Human; Ruffolo, R. R., Ed.; CRC: Boca Raton, FL, 1995; p 79; (e) Doherty, A. M. Drug Discov. Today 1996, 1, 60; (f) Webb, M. L.; Meek, T. D. Med. Res. Rev. 1997, 17, 17; (g) Liu, G. Annu. Rep. Med. Chem. 2000, 35, 73.

7. (a) Boigegrain, R.; Maffrand, J. P. Thieno[2,3- $d]$ pyridazin-4ones and thieno[2,3- $d$ ]pyridazin-7-ones and their therapeutic use. Fr. Demande 1981, p 22; (b) Boigegrain, R.; Maffrand, J. P. Thieno[2,3- $d]$ pyridazines and their therapeutic application. Fr. Demande 1981, p 18.

8. (a) Hood, H. E. Process for producing phenol by acid-catalyzed cleavage of cumene hydroperoxide. U.S. Patent 1994, p 22; (b) Lewandowski, G.; Milchert, E. Przem. Chem. 2002, 81, 103; (c) Takai, T. Shokubai 2003, 45, 354; (d) Levin, M. E.; Gonzales, N. O.; Zimmerman, L. W.; Yang, J. J. Hazard. Mater. 2006, 130,88 . 